\title{
Prolonged distal motor latency of median nerve does not improve diagnostic accuracy for CIDP
}

\author{
Emanuele Spina ${ }^{1}$ (1) Pietro Emiliano Doneddu ${ }^{2} \cdot$ Giuseppe Liberatore $^{2} \cdot$ Dario Cocito $^{3} \cdot$ Raffaella Fazio $^{4}$. \\ Chiara Briani $^{5} \cdot$ Massimiliano Filosto $^{6} \cdot$ Luana Benedetti $^{7,8}$. Giovanni Antonini ${ }^{9} \cdot$ Giuseppe Cosentino $^{10,11}$. \\ Stefano Jann ${ }^{12} \cdot$ Anna Mazzeo $^{13}$. Andrea Cortese ${ }^{11,14}$. Girolama Alessandra Marfia ${ }^{15}$. Angelo Maurizio Clerici ${ }^{16}$. \\ Gabriele Siciliano ${ }^{17}$. Marinella Carpo ${ }^{18}$ - Marco Luigetti ${ }^{19,20}$. Giuseppe Lauria ${ }^{21,22}$. Tiziana Rosso ${ }^{23}$. \\ Guido Cavaletti ${ }^{24}$. Erdita Peci ${ }^{3}$ - Stefano Tronci ${ }^{4} \cdot$ Marta Ruiz $^{5}$. Stefano Cotti Piccinelli ${ }^{6}$. Angelo Schenone ${ }^{7}$. \\ Luca Leonardi $^{9}$. Luca Gentile $^{13}$ - Laura Piccolo ${ }^{11}$. Giorgia Mataluni ${ }^{15}$. Lucio Santoro ${ }^{1}$. Eduardo Nobile-Orazio ${ }^{2,25}$. \\ Fiore Manganelli ${ }^{1}$ on behalf of The Italian CIDP Database Study Group
}

Received: 20 April 2021 / Revised: 14 June 2021 / Accepted: 14 June 2021 / Published online: 26 June 2021

(c) The Author(s) 2021, corrected publication 2021

\begin{abstract}
Compression of the median nerve at the carpal tunnel can give demyelinating features and result in distal motor latency (DML) prolongation fulfilling the EFNS/PNS demyelinating criteria for chronic inflammatory demyelinating polyneuropathy (CIDP). Accordingly, being carpal tunnel syndrome (CTS) common in the general population, the EFNS/PNS guidelines recommend excluding the DML of the median nerve when DML prolongation may be consistent with median neuropathy at the wrist from CTS. The main aims of this study were to verify whether the inclusion of DML of the median nerve (when consistent with CTS) could improve electrophysiological diagnostic accuracy for CIDP and if the median nerve at the carpal tunnel was more prone to demyelination. We analyzed electrophysiological data from 499 patients included consecutively into the Italian CIDP Database. According to the EFNS/PNS criteria, 352 patients had a definite, 10 a probable, and 57 a possible diagnosis of CIDP, while 80 were not fulfilling the diagnostic criteria. The inclusion of DML prolongation of median nerve did not improve significantly the diagnostic accuracy for CIDP; overall diagnostic class changed in 6 out of 499 patients (1.2\%) and electrodiagnostic class of CIDP changed from not fulfilling to possible in only 2 patients (2.5\% of not-fulfilling patients). In conclusion, we can infer that excluding DML prolongation of median nerve does not increase the risk of missing a diagnosis of CIDP thus corroborating the current EFNS/PNS criteria.
\end{abstract}

Keywords CIDP · Neurophysiology $\cdot$ Carpal tunnel syndrome $\cdot$ Diagnostic accuracy $\cdot$ Demyelination

\section{Introduction}

Carpal tunnel syndrome (CTS) is the most common nerve entrapment neuropathy worldwide [1]. It is due to compression of the median nerve in the narrow passage from the forearm to the hand, constituted by flexor retinaculum and bones of the wrist. CTS has a prevalence of 50 cases per

Statistical analysis was performed by Spina E.

Members of the Italian CIDP Database Study Group are listed in the Acknowledgement section at the end of the article.

Emanuele Spina

ema.spina@libero.it

Extended author information available on the last page of the article
1000 people, has female predominance and has an onset in the fifth or sixth decade [1]. The diagnosis of CTS is based on the clinical presentation of relevant symptoms and supported by electrophysiological findings due to the entrapment of the median nerve at the wrist. Distal motor latency (DML) prolongation along with sensory nerve conduction velocity (SNCV) slowing or absence of sensory action potential (SAP) is the hallmark of the electrophysiological diagnosis [2].

Chronic inflammatory demyelinating polyneuropathy (CIDP) is the most common acquired chronic inflammatory neuropathy and its prevalence range from 0.8 to 8.9 cases per 100,000 people.[3]. Diagnosis of CIDP relies on clinical signs and symptoms confirmed by electrophysiological test demonstrating peripheral nerve demyelination, according 
to the European Federation of Neurological Societies and Peripheral Nerve Society (EFNS/PNS) criteria. Supportive criteria can assist the diagnosis $[4,5]$.

DML prolongation $\geq 50 \%$ above the upper limit of normal (ULN) is a major EFNS/PNS electrophysiological criterion for diagnosing CIDP [4]. However, compression or entrapment itself (e.g. CTS) can give demyelinating features and result in DML prolongation fulfilling EFNS/PNS demyelinating criteria.

Accordingly, the EFNS/PNS guidelines recommend excluding the DML of the median nerve when DML prolongation may be consistent with median neuropathy at the wrist from CTS, that is if DML is associated with SNCV slowing or absence of SAP [4].

Under these premises, the main aim of this study was to verify whether the inclusion of DML of the median nerve (when consistent with CTS) could improve electrophysiological diagnostic accuracy for CIDP.

Moreover, since previous reports on small CIDP samples have not observed more severe demyelination at entrapment sites $[6,7]$ we decided to verify whether the median nerve at the carpal tunnel was more prone to demyelination in a larger CIDP population.

\section{Methods}

This was a retrospective multicentre cohort study in a large sample of CIDP patients implementing a web database (CINECA, Bologna, Italy) to collect demographical, clinical, and electrophysiological data from patients diagnosed and followed by 22 centers throughout Italy with expertise on CIDP.

The Ethical Committee of each participating Center approved the study and all the patients gave written informed consent. Experienced neurologists with a neuromuscular subspecialty obtained clinical and neurophysiological data. Verification of the diagnostic data for all of the enrolled patients was centralized in the database coordinator center (Humanitas). Data monitoring included diagnosis revision, suspect double entries, missing data, and plausibility checks. Patients with an alternative diagnosis for the neuropathy, an increased titers of anti-myelin-associated glycoprotein IgM antibodies or without available nerve conduction studies were excluded [8].

Based on electrophysiological findings, patients were classified according to EFNS/PNS guidelines in four CIDP diagnostic classes: definite, probable, possible, and not fulfilling. The reasons why patients who did not meet the criteria were equally diagnosed as CIDP was discussed in a recent paper. To note these patients had similar clinical features and frequency of abnormal supportive criteria for the diagnosis of CIDP compared to patients fulfilling EFNS/ PNS criteria [9], and we compared demographic features among these classes.

For the aim of this study, we first calculated the number of median nerves showing prolonged DML $(\geq 50 \%$ above ULN according to EFNS/PNS criteria) associated with SNCV slowing or absent SAP (e.g. consistent with CTS). Hence, we evaluated if the inclusion of prolonged DML changed significantly the proportion of electrophysiological diagnostic class of CIDP (from possible to definite, from not fulfilling to possible or definite) with multiple tests of proportions.

Afterward, we tested whether the median nerve might exhibit more severe demyelination at the carpal tunnel compared to the ulnar nerve at the wrist or and the same median nerve at the forearm. To do this, we compared the proportion of prolonged DML ( $\geq 50 \%$ above ULN) of median nerves (independently from the presence of sensory nerve conduction abnormalities) with the proportion of prolonged DML ( $\geq 50 \%$ above ULN) of ulnar nerves using a Chi-square test. As well, we compared both for median and ulnar nerves the proportion of electrophysiological abnormalities fulfilling EFNS/PNS criteria observed distally with the proportion of those found more proximally [i.e. motor nerve conduction velocity $(\mathrm{MNCV}) \geq 30 \%$ below the lower limit of normal (LLN) along the forearm] using Chi-square test. Moreover, using regression analysis, we evaluated the relationship between DML of median and ulnar nerves and between DML and MNCV of both median and ulnar nerves. Lastly, we evaluated if the proportion of prolonged DML varied between diagnostic classes and if there were differences in temporal dispersion. STATA 13 was used for statistical analysis.

\section{Results}

We analyzed data from 499 (out of 545) patients included consecutively into the Italian CIDP Database from January 2015 to January 2019. Forty-four patients were excluded from analysis due to the presence of a different cause for neuropathy, the unavailability of neurophysiological data, or the diagnosis (two patients) of chronic immune sensory polyradiculopathy (CISP) [10].

According to the EFNS/PNS criteria, 352 patients had a definite diagnosis of CIDP, 10 a probable diagnosis, 57 a possible diagnosis and 80 were not fulfilling the diagnostic criteria. There were no differences among diagnostic classes in age, gender and disease duration.

The total number of tested nerves (median and ulnar nerves) recorded in our database was 998 , including motor conduction of 592 median nerves and 656 ulnar nerves, and sensory conduction of 521 median and 656 ulnar nerves. 
Prolongation of DML fulfilling the EFNS/PNS criteria ( $\geq 50 \%$ above ULN) was found in $160(27 \%)$ out of 592 median nerves. In 43 (27\%) out of 160 median nerves sensory conduction was not performed, in 55/160 (34.3\%) SNCV was reduced and in 53/160 (33.1\%) SAP was absent.

Prolongation of DML without sensory conduction abnormalities was detected in only 9 (5.6\%) median nerves. Patients with prolonged DML of median nerve had a median age of 56 years and a male to female ratio of 1.7:1 comparable to median age (56 years) and male to female ratio (1.8:1) of our CIDP population as a whole.

Prolongation of DML fulfilling the EFNS/PNS criteria ( $\geq 50 \%$ above ULN) was found in $141(21.5 \%$ ) out of 656 ulnar nerves. In 41 (29\%) out of 141 ulnar nerves sensory conduction was not performed, in 44/141 (31.2\%) SNCV was reduced and in 48/141 (34\%) SAP was absent. Prolongation of DML without sensory conduction abnormalities was detected in only $8(5.6 \%)$ ulnar nerves.

With the inclusion of the 108 median nerves that showed DML prolongation fulfilling the EFNS/PNS criteria and consistent with CTS for electrophysiological diagnosis of CIDP, 4 patients moved from possible to definite electrophysiological diagnostic class of CIDP and 2 patients moved from not fulfilling to possible diagnostic class; no patient moved from not fulfilling to definite diagnostic class. Statistical analysis proved that the inclusion of the 108 prolonged DML values did not change significantly the diagnostic electrophysiological class of CIDP (Table 1).

The statistical analysis evaluating whether median nerves might exhibit more severe demyelination at carpal tunnel showed that the proportion of DML prolongation was comparable between median and ulnar nerves $(27 \%$ vs $21.5 \%$; $p=\mathrm{NS})$. Furthermore, MNCV reduction ( $\geq 30 \%$ below LLN) was found in 225 (38\%) out of 592 median nerves and in 232 (35.4\%) out of 656 ulnar nerves, disclosing a significantly higher proportion of demyelinating findings in the forearm than distal tract in both median (38\% vs $27 \%$; $p=0.02)$ and ulnar nerves $(35.4 \%$ vs $21.5 \%, p<0.001)$.

Linear regression analysis showed that DML in median nerve was directly related to DML in ulnar nerve and linear regression analysis showed that DML was inversely related to MNCV calculated in forearm both in median $(t:-8.09$, $p<0.001)$ and ulnar $(t:-10.71 ; p<0.001)$ nerves. We did not found any differences in the rate of prolonged DML nor temporal dispersion between diagnostic classes.

\section{Discussion}

A primary concern of clinicians facing a suspected CIDP patient is to miss the diagnosis and to delay access to proper treatment. This led to develop several diagnostic criteria for CIDP [11]. EFNS/PNS criteria have the best combination of sensitivity and specificity but about one-fourth of patients may not satisfy electrodiagnostic criteria (EFNS/PNS sensitivity $=73 \%$ ). Thus, clinical and supportive criteria play a major role in clinical practice in diagnosing CIDP when EFNS/PNS electrophysiological criteria are not satisfied [9].

Accordingly, we wondered if the inclusion of DML latency prolongation of median nerve could improve the diagnostic accuracy especially in the subset of patients not fulfilling the EFNS/PNS electrophysiological criteria.

We found that the inclusion of DML prolongation of median nerve does not improve significantly the diagnostic accuracy for CIDP. Indeed, DML inclusion overall changed diagnostic class in $6(1.2 \%)$ out of 499 patients and electrodiagnostic class of CIDP changed from not fulfilling to possible in only 2 patients ( $2.5 \%$ of not-fulfilling patients).

Anyway, DML prolongation fulfilling EFNS/PNS criteria was quite common in our cohort of CIDP patients being present in about one-fourth of tested median nerves (27\%).

Nevertheless, the statistical analysis did not reveal a higher proportion of abnormalities in the distal tract of median nerves rather than in ulnar nerves ( $27 \%$ vs $21.5 \%$ ) as well distal slowing in median nerves was parallel to the distal slowing in ulnar nerves. To note, the male to female ratio of CIDP patients with median nerve electrophysiological findings consistent with CTS fully matched that of the entire cohort of CIDP patients, not supporting superimposed CTS.

Moreover, in median nerves DML prolongation was related to MNCV slowing, as well we did not observe a higher

Table 1 Different frequencies of patient allocation in CIDP diagnostic classes considering and not considering electrophysiological criterion of prolonged DML ( $\geq 50 \%$ of upper limit of normal) of the median nerve consistent with CTS

\begin{tabular}{lll}
\hline & Standard EFNS/PNS criteria $(\%)$ & $\begin{array}{l}\text { EFNS/PNS criteria with prolonged DML of the median } \\
\text { nerve included }(\%)\end{array}$ \\
\hline Definite & $352 / 499(70.6)$ & $356 / 499(71.4)$ \\
Probable & $10 / 499(2)$ & $10 / 499(2)$ \\
Possible & $57 / 499(11.4)$ & $55 / 499(11)$ \\
Not fulfilling & $80 / 499(16)$ & $78 / 499(15.6)$ \\
\hline
\end{tabular}

CTS carpal tunnel syndrome, $D M L$ distal motor latency

*Chi-square test for multiple proportion comparison 
proportion of electrophysiological abnormalities fulfilling EFNS/PNS criteria at the wrist rather than to the forearm (i.e. prolonged DML vs reduced MNCV). These electrophysiological findings were similarly found in ulnar nerves hence pointing up a high concordance of demyelinating electrodiagnostic features between the upper limb nerves [12].

Taken together, our data suggest that demyelination that occurs distally in the median nerve generally follows a more widespread demyelinating process. This observation is in line with a previous description in literature, in which the comparison between CIDP and CTS patients showed a higher dCMAP duration and a higher duration/distal latency ratio in CIDP patients, a sign of broader demyelination [13]. Accordingly, our findings from a very large cohort of patients confirm that the median nerve at the carpal tunnel is not more prone to demyelination in line with previous reports in smaller samples of CIDP patients [6, 7].

In conclusion, we can infer that excluding DML prolongation of the median nerve does not increase the risk of missing a diagnosis of CIDP thus corroborating the current EFNS/PNS criteria.

Acknowledgements Italian CIDP Database Study Group: Pietro Emiliano Doneddu, Giuseppe Liberatore, Francesca Gallia, and Eduardo Nobile-Orazio from the Department of Medical Biotechnology and Translational Medicine, Neuromuscular and Neuroimmunology Service, Humanitas Clinical and Research Institute, Milan University; Rozzano, Milan, Italy; Erdita Peci and Dario Cocito from the Department of Neuroscience, University of Turin, Turin, Italy. Stefano Tronci and Raffaella Fazio from the Division of Neuroscience, Department of Neurology, Institute of Experimental Neurology (INSPE), San Raffaele Scientific Institute, Milan, Italy; Fiore Manganelli, Lucio Santoro, Emanuele Spina and Enrica Pisano from the Department of Neuroscience, Reproductive Sciences and Odontostomatology, University of Naples 'Federico II', Naples, Italy; Marta Ruiz and Chiara Briani from the Neurology Unit, Department of Neuroscience, University of Padua, Padua, Italy. Stefano Cotti Piccinelli and Massimiliano Filosto from the Center for Neuromuscular Diseases and Neuropathies, Unit of Neurology ASST 'Spedali Civili', University of Brescia, Brescia, Italy; Alessandro Beronio and Luana Benedetti from the Neurology Unit, Sant'Andrea Hospital, La Spezia, Italy; Antonio Toscano, Luca Gentile and Anna Mazzeo from the Department of Clinical and Experimental Medicine, Unit of Neurology, University of Messina, Messina, Italy; Giorgia Mataluni and Girolama Alessandra Marfia from the Disimmune Neuropathies Unit, Department of Systems Medicine, Tor Vergata University of Rome, Rome, Italy; Laura Piccolo, Elisa Vegezzi and Andrea Cortese from the IRCCS Foundation C. Mondino National Neurological Institute, Pavia, Italy; Giuseppe Cosentino and Brigida Fierro from the Department of Experimental BioMedicine and Clinical Neurosciences (BioNeC), University of Palermo, Palermo, Italy; Verrengia Elena Pinuccia and Stefano Jann from the Department of Neuroscience, Niguarda Ca' Granda Hospital, Milan, Italy; Elisa Bianchi and Ettore Beghi from the Laboratorio di Malattie Neurologiche, IRCCSIstituto Mario Negri, Milan, Italy; Angelo Maurizio Clerici from the Neurology Unit, Circolo \& Macchi Foundation Hospital, Insubria University, DBSV, Varese, Italy; Federica Scrascia and Marinella Carpo from the ASST Bergamo Ovest-Ospedale Treviglio, Treviglio, Italy; Martina Garnero and Angelo Schenone from the Department of Neuroscience, Rehabilitation, Ophthalmology, Genetics, Maternal and Child Health, University of Genoa and IRCCS AOU San Martino-IST,
Genoa, Italy; Marco Luigetti and Mario Sabatelli from the Department of Neurology, Catholic University of Sacred Heart, Rome, Italy; Patrizia Dacci and Giuseppe Lauria from the Unit of Neuroalgology, IRCCS Foundation "Carlo Besta" Neurological Institute, Milan, Italy; Luca Leonardi and Giovanni Antonini from the Unit of Neuromuscular Diseases, Department of Neurology Mental Health and Sensory Organs (NESMOS), Faculty of Medicine and Psychology, 'Sapienza' University of Rome, Sant'Andrea Hospital, Rome, Italy; Tiziana Rosso from the Azienda UL.SS. 8 Asolo, Castelfranco Veneto, Italy; Erika Schirinzi and Gabriele Siciliano from the Neurology Unit, Department of Clinical and Experimental Medicine, University of Pisa, Pisa, Italy; Claudia Balducci and Guido Cavaletti from the School of Medicine and Surgery and Experimental Neurology Unit, University of MilanoBicocca, Monza, Italy.

PietroEmiliano Doneddu, Giuseppe Liberatore, Francesca Gallia, Eduardo Nobile-Orazio, Rozzano, Erdita Peci, Dario Cocito, Stefano Tronci, Raffaella Fazio, San Raffaele, Fiore Manganelli, Lucio Santoro, Emanuele Spina, Enrica Pisano, Marta Ruiz, Chiara Briani, StefanoCotti Piccinelli, Massimiliano Filosto, Alessandro Beronio, Luana Benedetti, Antonio Toscano, Luca Gentile, Anna Mazzeo, Giorgia Mataluni, GirolamaAlessandra Marfia, Laura Piccolo, Elisa Vegezzi, Andrea Cortese, Giuseppe Cosentino, Brigida Fierro, VerrengiaElena Pinuccia, Stefano Jann, Elisa Bianchi, Ettore Beghi, AngeloMaurizio Clerici, Federica Scrascia, Marinella Carpo, Martina Garnero, Angelo Schenone, Marco Luigetti, Mario Sabatelli, Patrizia Dacci, Giuseppe Lauria, Luca Leonardi, Giovanni Antonini, Tiziana Rosso, Rika Schirinziand Gabriele Siciliano, Claudia Balducci, Guido Cavaletti

Funding Open access funding provided by Università degli Studi di Napoli Federico II within the CRUI-CARE Agreement.

Data availability The data supporting the findings of this study are available on request from the corresponding author.

\section{Declarations}

Conflict of interest P.E.D. reported travel Grants to attend scientific meetings from CSL Behring and Kedrion. G.L. reported travel Grants to attend scientific meetings from CSL Behring and Kedrion. D.C. reported honoraria for lecturing from Shire, CSL Behring, and Kedrion and travel Grants to attend scientific meeting from Shire, Kedrion, and CSL Behring. R.F. has served on scientific advisory boards for CSL Behring and has received travel Grants from Kedrion and CSL Behring to attend scientific meeting. C.B. has served on scientific advisory boards for Pfizer and has received travel Grants from Kedrion and CSL Behring to attend scientific meeting. M.F. has served on scientific advisory boards for CSL Behring and Sarepta Therapeutics and has received travel Grants from Sanofi Genzyme, Kedrion, Baxter and CSL Behring to attend scientific meeting. G.A. reported honoraria for lecturing from Kedrion and Sanofi-genzyme, travel Grants from Kedrion, Sanofi-Genzyme and LJ Pharma. G.C. reported travel Grants to attend scientific meetings from CSL Behring and Kedrion. S.J. reported research Grants from Grifols, outside this work, and travel Grants from Grifols and Kedrion. A.M. reported travel Grants from Kedrion and CSL Behring to attend scientific meeting. A.C. reported travel Grants to attend scientific meetings from Kedrion. G.A.M. reported consulence fees and travel fundings from CSL Behring, Kedrion, Shire and Grifols. M.C. reported travel Grants to attend scientific meetings from Kedrion. M.L. reported honoraria for scientific board from Pfeizer and Alnylam and travel Grants from Pfeizer, Grifols and Kedrion to attend scientific meeting. G.C. reported honoraria for lecturing and travel Grants to attend scientific meetings from Kedrion. E.P. reported travel Grants to attend scientific meetings from CSL Behring. L.G. reported travel Grants from Kedrion and CSL Behring to attend scien- 
tific meeting. G.M. reported consulence fees and travel fundings from CSL Behring, Kedrion, Shire and Grifols. L.S. reported personal fees for scientific events from CSL Behring and has received travel Grants to attend scientific meetings from CSL Behring and Kedrion. E.N.O. reported personal fees for Advisory or Scientific Board from Kedrion, Italy, Baxter, Italy, Novartis, Switzerland, CSL-Behring, Italy, LFB, France, Astellas, the Netherlands, outside the submitted work and travel Grants to attend Scientific Meeting from Baxter, Grifols, Kedrion, and Novartis, Italy. F.M. reported personal fees for scientific events from CSL Behring and has received travel Grants to attend scientific meetings from CSL Behring and Kedrion. The other authors declare no conflict of interest.

Ethics approval The study was approved by the ethical committee of each participating center.

Consent to participate Written informed consent was obtained from all participants for enrolment.

Open Access This article is licensed under a Creative Commons Attribution 4.0 International License, which permits use, sharing, adaptation, distribution and reproduction in any medium or format, as long as you give appropriate credit to the original author(s) and the source, provide a link to the Creative Commons licence, and indicate if changes were made. The images or other third party material in this article are included in the article's Creative Commons licence, unless indicated otherwise in a credit line to the material. If material is not included in the article's Creative Commons licence and your intended use is not permitted by statutory regulation or exceeds the permitted use, you will need to obtain permission directly from the copyright holder. To view a copy of this licence, visit http://creativecommons.org/licenses/by/4.0/.

\section{References}

1. Padua L, Coraci D, Erra C et al (2016) Carpal tunnel syndrome: clinical features, diagnosis, and management. Lancet Neurol 15:1273-1284. https://doi.org/10.1016/S1474-4422(16)30231-9

2. Padua L, Lo Monaco M, Gregori B, Valente EM, Padua R, Tonali $P$ (1997) Neurophysiological classification and sensitivity in 500 carpal tunnel syndrome hands. Acta Neurol Scand 96:211-217

3. Querol L, Crabtree M, Herepath M, Priedane E, Viejo Viejo I, Agush S, Sommerer P (2020) Systematic literature review of burden of illness in chronic inflammatory demyelinating polyneuropathy (CIDP). J Neurol. https://doi.org/10.1007/ s00415-020-09998-8
4. Van der Bergh PYK, Hadden RDM, Bouche P, Cornblath DR, Hahn A, Illa I, Koski CL, Leger JM, Nobile-Orazio E, Pollard L, Sommer C, van Doorn PA, van Schaik IN (2010) European Federation of Neurological Societies/Peripheral Nerve Society Guideline on management of chronic inflammatory demyelinating polyradiculoneuropathy: report of a joint task force of the European Federation of Neurological Societies and the Peripheral Nerve Society-first revision. Eur J Neurol 17:356-363. https:// doi.org/10.1111/j.1468-1331.2009.02930.x

5. Spina E, Doneddu PE, Liberatore G, Italian CIDP Database Study Group et al (2021) The neurophysiological lesson from the Italian CIDP database. Neurol Sci. https://doi.org/10.1007/s10072-02105321-z (PMID: 34021439)

6. Padua L, Caliandro P, Aprile I, Sabatelli M, Madia F, Tonali P (2004) Occurence of nerve entrapment lesion in chronic inflammatory demyelinating polyneuropathy. Clin Neurophysiol 115:1140-1144. https://doi.org/10.1016/j.clinph2003.12.007

7. Rajabally YA, Narasimhan M (2011) Electrophysiological entrapment syndromes in chronic inflammatory demyelinating polyneuropathy. Muscle Nerv 44:444-447

8. Liberatore G, Manganelli F, Cocito D, Italian CIDP Database Study Group et al (2020) Relevance of diagnostic investigations in chronic inflammatory demyelinating poliradiculoneuropathy: data from the Italian CIDP database. J Peripher Nerv Syst. https:// doi.org/10.1111/jns. 12378

9. Liberatore G, Manganelli F, Cocito D, Italian CIDP Database Study Group et al (2021) Chronic inflammatory demyelinating poliradiculoneuropathy: can a diagnosis be made in patients not fulfilling electrodiagnostic criteria? Eur J Neurol 28(2):620-629. https://doi.org/10.1111/ene.14545 (Epub 2020 Oct 15 PMID: 32959475)

10. Nobile-Orazio E (2014) Chronic inflammatory demyelinating polyradiculoneuropathy and variants: where we are and where we should go. J Periph Nerv Syst 19(1):2-13. https://doi.org/10. 1111/jns5.12053 (PMID:14612201 Review)

11. Breiner A, Brannagan TH 3rd (2014) Comparison of sensitivity and specificity among 15 criteria for chronic inflammatory demyelinating polyneuropathy. Muscle Nerve 50:40-46. https://doi.org/ 10.1002 /mus. 24088

12. Kuwabara S, Ogawara K, Misawa S, Mori M, Hattori T (2002) Distribution patterns of demyelination correlate with clinical profiles in chronic inflammatory demyelinating polyneuropathy. J Neurol Neurosurg Psychiatry 72:37-42. https://doi.org/10.1136/ jnnp.72.1.37

13. Cleland JC, Logigian EL, Pariwat T, Herrmann DB (2003) Dispersion of the distal compound muscle action potential in chronic inflammatory demyelinating polyneuropathy and carpal tunnel syndrome. Muscle Nerv 28(2):189-193. https://doi.org/10.1002/ mus. 10420

\title{
Authors and Affiliations
}

\author{
Emanuele Spina ${ }^{1}$ (D) Pietro Emiliano Doneddu ${ }^{2}$ - Giuseppe Liberatore ${ }^{2} \cdot$ Dario Cocito $^{3} \cdot$ Raffaella Fazio $^{4}$. \\ Chiara Briani ${ }^{5}$. Massimiliano Filosto ${ }^{6} \cdot$ Luana Benedetti $^{7,8}$. Giovanni Antonini ${ }^{9}$. Giuseppe Cosentino ${ }^{10,11}$. \\ Stefano Jann ${ }^{12}$. Anna Mazzeo ${ }^{13}$. Andrea Cortese ${ }^{11,14}$. Girolama Alessandra Marfia ${ }^{15}$. Angelo Maurizio Clerici ${ }^{16}$. \\ Gabriele Siciliano $^{17}$. Marinella Carpo ${ }^{18} \cdot$ Marco Luigetti $^{19,20}$. Giuseppe Lauria ${ }^{21,22}$. Tiziana Rosso ${ }^{23}$. \\ Guido Cavaletti ${ }^{24}$. Erdita Peci ${ }^{3}$. Stefano Tronci ${ }^{4}$ Marta Ruiz ${ }^{5}$. Stefano Cotti Piccinelli ${ }^{6}$. Angelo Schenone ${ }^{7}$. \\ Luca Leonardi ${ }^{9}$. Luca Gentile ${ }^{13}$ - Laura Piccolo ${ }^{11}$. Giorgia Mataluni ${ }^{15}$ - Lucio Santoro ${ }^{1}$. Eduardo Nobile-Orazio ${ }^{2,25}$. \\ Fiore Manganelli ${ }^{1}$ on behalf of The Italian CIDP Database Study Group
}


1 Department of Neuroscience, Reproductive Sciences and Odontostomatology, University of Naples "Federico II", Via Pansini, 5, 81025 Naples, Italy

2 Neuromuscular and Neuroimmunology Service, IRCCS Humanitas Clinical and Research Institute, Rozzano, Milan, Italy

3 Presidio Sanitario Major, Istituti Clinici Scientifici Maugeri, Turin, Italy

4 Division of Neuroscience, Department of Neurology, Institute of Experimental Neurology (INSPE), San Raffaele Scientific Institute, Milan, Italy

5 Department of Neuroscience, Neurology Unit, University of Padova, Padova, Italy

6 Center for Neuromuscular Diseases and Neuropathies, Unit of Neurology, ASST 'Spedali Civili', University of Brescia, Brescia, Italy

7 Department of Neuroscience, Rehabilitation, Ophthalmology, Genetics, Maternal and Child Health, University of Genoa and IRCCS San Martino, Genoa, Italy

8 IRCCS AOU San Martino-IST, Genoa, Italy

9 Unit of Neuromuscular Diseases, Department of Neurology Mental Health and Sensory Organs (NESMOS), Faculty of Medicine and Psychology, Sant'Andrea Hospital, 'Sapienza' University of Rome, Rome, Italy

10 Department of Brain and Behavioral Sciences, University of Pavia, Pavia, Italy

11 IRCCS Mondino Foundation, Pavia, Italy

12 Department of Neuroscience, Niguarda Ca' Granda Hospital, Milan, Italy
13 Department of Clinical and Experimental Medicine, Unit of Neurology, University of Messina, Messina, Italy

14 Molecular Neurosciences, University College London, London, UK

15 Dysimmune Neuropathies Unit, Department of Systems Medicine, Tor Vergata University of Rome, Rome, Italy

16 Neurology Unit, Circolo and Macchi Foundation Hospital, Insubria University, DBSV, Varese, Italy

17 Neurology Unit, Department of Clinical and Experimental Medicine, University of Pisa, Pisa, Italy

18 Neurology Unit, ASST Bergamo Ovest-Ospedale Treviglio, Treviglio, Italy

19 UOC Neurologia, Fondazione Policlinico Universitario Agostino Gemelli IRCCS, Rome, Italy

20 Dipartimento di Neuroscienze, Università Cattolica del Sacro Cuore, Rome, Italy

21 Unit of Neuroalgology, IRCCS Foundation 'Carlo Besta' Neurological Institute, Milan, Italy

22 Department of Biomedical and Clinical Sciences 'Luigi Sacco', University of Milan, Milan, Italy

23 ULSS2 Marca Trevigiana, UOC Neurologia-Castelfranco Veneto, Treviso, Italy

24 School of Medicine and Surgery and Experimental Neurology Unit, University of Milano-Bicocca, Monza, Italy

25 Department of Medical Biotechnology and Translational Medicine, Milan University, Milan, Italy 